\title{
Composition of the pericellular matrix modulates the deformation behaviour of chondrocytes in articular cartilage under static loading
}

\author{
Petro Julkunen · Wouter Wilson · Jukka S. Jurvelin • \\ Rami K. Korhonen
}

Received: 7 April 2009/Accepted: 22 October 2009/Published online: 7 November 2009

(c) The Author(s) 2009. This article is published with open access at Springerlink.com

\begin{abstract}
The aim was to assess the role of the composition changes in the pericellular matrix (PCM) for the chondrocyte deformation. For that, a three-dimensional finite element model with depth-dependent collagen density, fluid fraction, fixed charge density and collagen architecture, including parallel planes representing the split-lines, was created to model the extracellular matrix (ECM). The PCM was constructed similarly as the ECM, but the collagen fibrils were oriented parallel to the chondrocyte surfaces. The chondrocytes were modelled as poroelastic with swelling properties. Deformation behaviour of the cells was studied under $15 \%$ static compression. Due to the depth-dependent structure and composition of cartilage, axial cell strains were highly depth-dependent. An increase in the collagen content and fluid fraction in the PCMs increased the lateral cell strains, while an increase in the fixed charge density induced an inverse behaviour. Axial cell strains were only slightly affected by the changes in PCM composition. We conclude that the PCM composition plays a significant role in the deformation behaviour
\end{abstract}

P. Julkunen $(\bowtie)$

Department of Clinical Neurophysiology, Kuopio University

Hospital, Kuopio, Finland

e-mail: petro.julkunen@kuh.fi

P. Julkunen - J. S. Jurvelin · R. K. Korhonen

Department of Physics, University of Kuopio, Kuopio, Finland

W. Wilson

Department of Biomedical Engineering, Eindhoven University of Technology, Eindhoven, The Netherlands

R. K. Korhonen

Human Performance Laboratory, Faculty of Kinesiology,

University of Calgary, Calgary, AB, Canada of chondrocytes, possibly modulating cartilage development, adaptation and degeneration. The development of cartilage repair materials could benefit from this information.

Keywords Articular cartilage · Finite element analysis · Chondrocyte $\cdot$ Pericellular matrix $\cdot$ Fibril-reinforced

\section{Introduction}

Chondrocytes are responsible for the biosynthesis and maintenance of articular cartilage constituents, i.e. collagen and proteoglycans (PGs) [1-3]. During osteoarthritis (OA), the balance of these constituents is disturbed due to changes of the anabolic and catabolic activities within chondrocytes [4]. Chondrocyte strains have been suggested to be related to the cartilage biosynthesis $[1,2,5]$. Hence, it is important to understand the influence of the mechanical environment of chondrocytes on the mechanical signals perceived by cells. The pericellular matrix (PCM, Fig. 1), which includes a shell of collagen fibres around the cells [6-8], as well as relatively high PG content $[6,9,10]$, can modify the deformation behaviour of chondrocytes [11-13]. The importance of the mechanical properties of the PCM for the cell deformations has been studied in a few studies [11-13]. However, the influence of the PCM composition (collagen, PGs and interstitial water) on the deformation of chondrocytes is currently poorly understood.

The three-dimensional (3D) collagen network of articular cartilage is highly organized [14]. The collagen network architecture has been implemented in macroscopic models of cartilage [15-20]. In the first theoretical studies on chondrocyte mechanics, a 3D collagen structure of the 


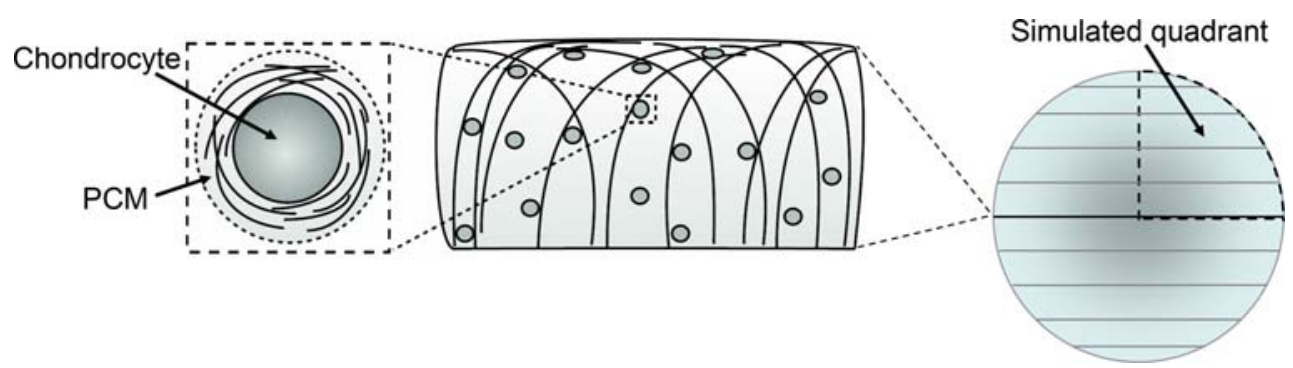

Fig. 1 Schematic 2D presentation of articular cartilage with collagen network and chondrocytes in $x y$-plane. One chondron with the pericellular collagen has been zoomed on the left. On the right, a top

cartilage tissue was not included [12, 21-25]. Our recent studies $[13,26]$ showed that the arcade-like fibril network as well as the collagen fibrils in the PCM significantly influenced chondrocyte deformation in a depth-dependent manner. However, the models of those studies were not able to estimate tissue function based on composition, and they neglected split-lines in the extracellular matrix (ECM).

Based on a recently presented macroscopic finite element (FE) model [27], which is based on a composition of biphasic structures, a microscopic model including the chondrocytes and PCMs was created in 3D including collagen split-lines in the ECM. This is the first attempt to combine a 3D macroscopic tissue-level model with a cellular-level model. With this model, the effects of collagen network, fixed charge density (FCD) and interstitial fluid of the PCM, as well as the effect of split-lines of the ECM on static deformations and stress-strain environment of the chondrocytes were studied. We hypothesized that the collagen fibrils and FCD of the PCM modulate cell deformations in an opposite manner, and that cells are deformed primarily along the collagen split-line direction of the ECM.

\section{Materials and methods}

\subsection{Fibril-reinforced biphasic swelling model}

A 3D mesh of a cartilage plug (height $0.75 \mathrm{~mm}$, diameter $3 \mathrm{~mm}$ ) was constructed for unconfined compression geometry [6] (Fig. 2). The mesh included three chondrocytes with the PCMs in different cartilage zones, located at the axis of symmetry. The chondrocytes were initially considered round with diameters of $10,14.5$ and $12 \mu \mathrm{m}$ in the superficial, middle and deep zones, respectively [6]. PCM thickness was $2.5,3.625$ and $3 \mu \mathrm{m}$ in the superficial, middle and deep zones, respectively [26, 28, 29]. A recently developed material with realistic depth-dependent structure and composition of articular cartilage (non-fibrillar part, view (xz-plane) of an articular cartilage sample is presented with horizontal lines representing the split-line directions. Simulated quadrant of the sample is indicated

fibrillar part, fluid) was applied for the PCMs and ECM [27]. Cells were modelled in a similar manner as the PCMs and ECM, except that they included no fibrillar part. The information of structural, compositional and mechanical properties of the cells, PCMs and ECM were obtained from the literature $[6,13,20,26,28-33]$.

The model is based on a standard biphasic theory:

$\sigma_{t o t}=-p I+\sigma_{s}=-n_{\mathrm{s}} p I-n_{\mathrm{f}} p I+\sigma_{s}$,

where $p$ is the hydrostatic pressure, $\boldsymbol{\sigma}_{\mathbf{s}}$ is the effective solid stress tensor, $\mathbf{I}$ is unit tensor, and $n_{\mathrm{f}}$ and $n_{\mathrm{s}}$ are the fluid and

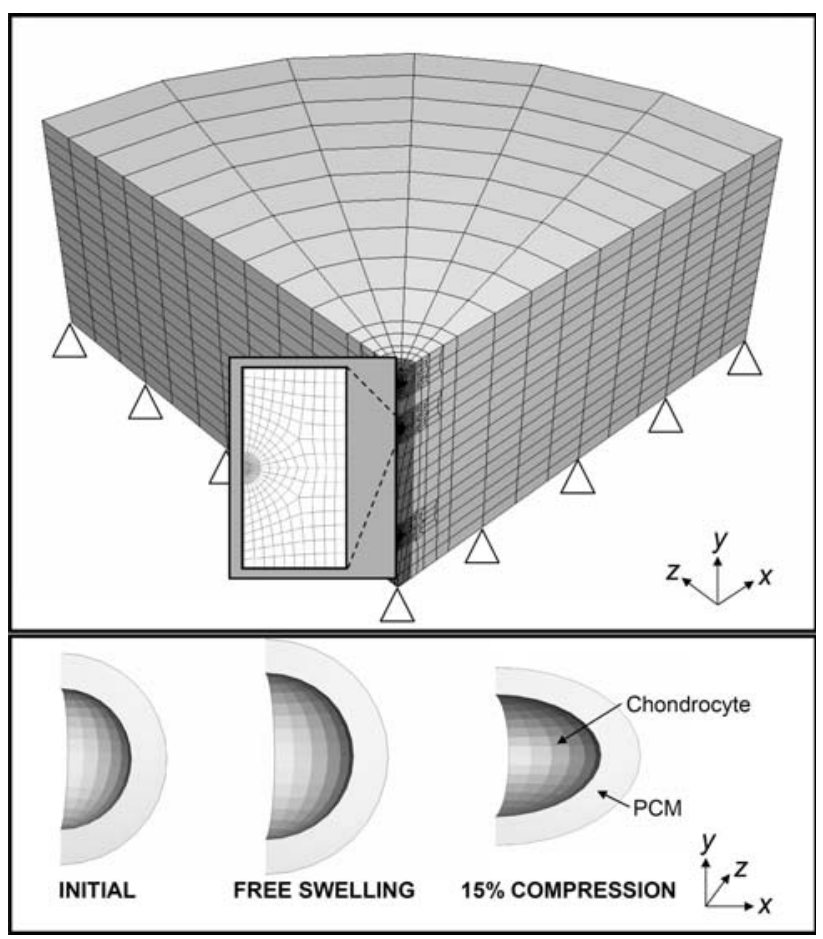

Fig. 2 Finite element mesh for 3D cartilage sample. One quadrant of the sample was modelled by applying symmetry conditions to both splitting ( $x y$ - and $z y$-plane) edges. Fluid was allowed to flow through the cartilage edge (and top during the swelling). Below the mesh, the deformation of the middle zone chondron is presented after each simulation step 
solid volume fractions, respectively. Differing from the original biphasic theory [34], the influence of changes in the solid volume fraction is accounted for in the model. Then, the total stress becomes:

$\sigma_{t o t}=-p I+n_{\mathrm{s}} \sigma_{s}=-p I+\frac{n_{\mathrm{s}, 0}}{J} \sigma_{r s}=-p I+n_{\mathrm{s}, 0} \sigma_{r s, J}(J)$,

where $n_{\mathrm{s}, 0}$ and $n_{\mathrm{s}}$ are the initial and current volume fractions of the solid matrix, $J$ is the volumetric deformation and $\sigma_{\mathbf{r s}}$ is the real solid stress tensor. After including the osmotic swelling, Eq. 2 becomes:

$\sigma_{t o t}=-\mu^{\mathrm{f}} I-\Delta \pi I+n_{\mathrm{s}, 0} \sigma_{r s, J}$,

where $\mu^{\mathrm{f}}$ is the water chemical potential and $\Delta \pi$ is the osmotic pressure difference $\left(p=\mu^{\mathrm{f}}+\Delta \pi\right)$. By separating the fibrillar and non-fibrillar part, Eq. 3 becomes:

$\sigma_{\text {tot }}=-\mu^{\mathrm{f}} I+n_{\mathrm{s}, 0}\left(\left(1-\sum_{i=1}^{\mathrm{totf}} \rho_{c}^{i}\right) \sigma_{n f}+\sum_{i=1}^{\mathrm{totf}} \rho_{c}^{i} \sigma_{f}^{i}\right)-\Delta \pi I$,

where $\rho_{\mathrm{c}}^{i}$ is the volume fraction of the collagen fibrils in $i$ th fibril with respect to the total volume of the solid matrix, $\boldsymbol{\sigma}_{\mathbf{n f}}$ is the stress tensor of the non-fibrillar matrix, $\boldsymbol{\sigma}_{\mathbf{f}}$ is the fibril stress tensor.

The stress of the non-fibrillar solid matrix can be described as:

$$
\begin{aligned}
\boldsymbol{\sigma}_{n f}= & \frac{2}{J} \mathbf{F} \frac{\partial W_{\mathrm{nf}}}{\partial \mathbf{C}} F^{T}=-\frac{1}{6} \frac{\ln (J)}{J} G_{\mathrm{m}} I \\
& {\left[-1+\frac{3\left(J+n_{\mathrm{s}, 0}\right)}{\left(-J+n_{\mathrm{s}, 0}\right)}+\frac{3 \ln (J)+J n_{\mathrm{s}, 0}}{\left(-J+n_{\mathrm{s}, 0}\right)^{2}}\right] } \\
& +\frac{G_{\mathrm{m}}}{J}\left(F \cdot F^{T}-J^{2 / 3} I\right),
\end{aligned}
$$

where $\boldsymbol{\sigma}_{\mathbf{n f}}$ is the Cauchy stress tensor of the non-fibrillar matrix, $G_{\mathrm{m}}$ is the shear modulus, $\mathbf{C}$ is the Cauchy-Green deformation tensor, $W_{\mathrm{nf}}$ is the energy function of the nonfibrillar matrix, and $J$ is the determinant of the deformation tensor $\mathbf{F}$ [20]. The shear modulus of the solid matrix in the PCM was chosen to be 10 times lower $(90 \mathrm{kPa})$, than that of the ECM $(900 \mathrm{kPa})[27,35]$. The solid matrix shear modulus of the cells was $17 \mathrm{kPa}$, which was assumed to account for all solid constituents of the cells as has also been assumed earlier [29]. Effective shear moduli depend on the water fraction of the matrix in the cells, PCMs and ECM.

The fibril stress tensor was given as follows [27]:

$\sigma_{f}=\frac{\lambda}{J} P_{\mathrm{f}} \vec{e}_{\mathrm{f}} \vec{e}_{\mathrm{f}}$,

where $\lambda$ is the elongation of the fibril, $P_{f}$ the 1st PiolaKirchhoff fibril stress, and $\vec{e}_{\mathrm{f}}$ the current fibril direction.
Mechanical behaviour of the collagen fibrils depends on the total fibril strain $\left(\varepsilon_{\mathrm{f}}\right)$, strain of the nonlinear spring $\left(\varepsilon_{\mathrm{e}}\right)$ and the values of $E_{1}, k_{1}, E_{2}, k_{2}$ and $n_{0}$ [27] (Fig. 3):

$P_{1}=E_{1}\left(e^{k_{1} \varepsilon_{\mathrm{f}}-1}\right)$

$P_{2}=E_{2}\left(e^{k_{2} \varepsilon_{\mathrm{e}}-1}\right)$

$P_{\mathrm{f}}=P_{1}+P_{2}$,

Values for $E_{1}$ and $k_{1}$ (describing the behaviour of the linear part of the collagen stiffness) were set to $4.316 \mathrm{MPa}$ and 16.85 , respectively [27]. Values for $E_{2}, k_{2}$ and $n_{0}$ (describing the behaviour of the nonlinear part of the collagen stiffness) for ECM were previously determined to be $19.97 \mathrm{MPa}, 41.49$ and $1.424 \times 10^{5} \mathrm{MPa}$ s, respectively [27]. For PCM (predominantly type VI) collagen, 10\% stiffness of the ECM (mostly type II) collagen stiffness was assumed in the reference configuration. This was justified by the geometrical differences in the collagen types, type II collagen being wider in diameter than type VI collagen [36].

The fibril structure was implemented according to Wilson et al. [37], including 2 primary and 7 secondary fibril directions. The density of each collagen fibril was presented as a function of the total collagen density:

$$
\rho_{c}=\rho_{c, \text { tot }} \frac{C}{2 C+7} \text { for primary fibrils, }
$$

$\rho_{c}=\rho_{c, \text { tot }} \frac{1}{2 C+7}$ for secondary fibrils,

where $C$ was 3.009 [37]. In the ECM, the collagen fibrils ran parallel to the articular surface in the superficial zone, bending in the middle zone towards perpendicular orientation in the deep zone. Thickness of superficial and middle zones were set to 10 and $40 \%$ of the total cartilage thickness, respectively [6]. Collagen fibrils were implemented in parallel planes in $x y$ direction representing the direction of split-lines in the ECM (Fig. 1). The collagen fibrils in the PCMs were orientated parallel to the cell surfaces (Fig. 1) $[7,8]$, and the cells included no collagen fibrils.

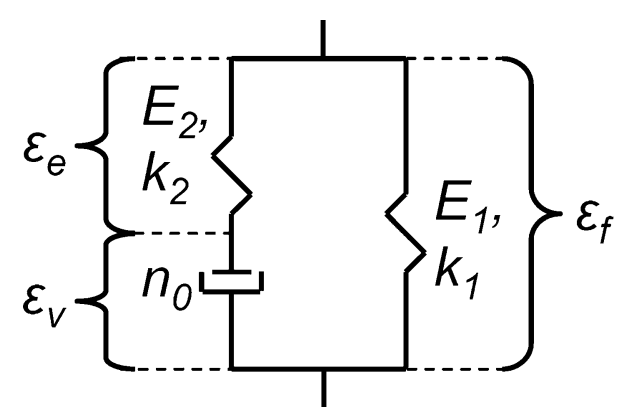

Fig. 3 Schematic spring/dashpot model for a viscoelastic collagen fibril. Material properties of the collagen fibril are described with the total fibril strain $\left(\varepsilon_{\mathrm{f}}\right)$, the strain of nonlinear spring $\left(\varepsilon_{\mathrm{e}}\right)$ and the values of model parameters $E_{1}, k_{1}, E_{2}, k_{2}$ and $n_{0}$ [27] 
The osmotic pressure gradient was implemented according to Wilson et al. [27]:

$\Delta \pi=\varphi_{\mathrm{int}} R T \sqrt{c_{\mathrm{F}}^{2}+4 \frac{\gamma_{\mathrm{ext}}^{ \pm^{2}}}{\gamma_{\mathrm{int}}^{ \pm^{2}}} c_{\mathrm{ext}}^{2}}-2 \varphi_{\mathrm{ext}} R T c_{\mathrm{ext}}$,

where $\varphi_{\text {int }}$ and $\varphi_{\text {ext }}$ are osmotic coefficients, $\gamma_{\text {int }}$ and $\gamma_{\text {ext }}$ are activity coefficients, and $c_{\text {ext }}$ is the external salt concentration $(0.15 \mathrm{M})$, and $c_{\mathrm{F}}$ is the FCD [38]. $R$ and $T$ are the gas constant $(8.3145 \mathrm{~J} / \mathrm{mol} \mathrm{K})$ and absolute temperature $(293 \mathrm{~K})$, respectively. The effective FCD, $c_{\mathrm{F}, \mathrm{eff}}$, takes the extra- and intra-fibrillar water into account:

$c_{\mathrm{F}, \mathrm{eff}}=\frac{n_{\mathrm{f}} c_{\mathrm{F}}}{n_{\mathrm{ext}}}$

where $n_{\text {ext }}$ is the volume fraction of the extra-fibrillar water as a function of the extra-fibrillar water mass fraction $\left(n_{\text {ext,m }}\right)$ :

$n_{\mathrm{ext}}=\frac{\rho_{\mathrm{s}} n_{\mathrm{ext}, \mathrm{m}}}{1-n_{\mathrm{ext}, \mathrm{m}}+\rho_{\mathrm{s}} n_{\mathrm{ext}, \mathrm{m}}}$

where $\rho_{\mathrm{s}}$ is the solid matrix mass density, which was set to $1.4338[39,40]$.

The permeability was implemented in the model according to Wilson et al. [20]:

$k=\alpha\left(1-n_{\mathrm{ext}}\right)^{-M}$,

where $\alpha$ and $M$ are positive material constants with the values of $1.767 \times 10^{-17} \mathrm{~m}^{4} / \mathrm{N} \mathrm{s}$ and 1.339 in the ECM, respectively [20]. In accordance with the literature [35], the permeability of the PCM was chosen to be the same as that of the ECM, while the permeability of the cells was fixed at $2.6 \times 10^{-12} \mathrm{~m}^{4} / \mathrm{N} \mathrm{s}$ [22]. A more thorough implementation of the constitutive laws into the FE-model was presented in a recent study by Wilson et al. [27].

Depth-dependent composition (distribution of FCD, collagen mass fraction and fluid fraction) of the ECM and PCMs were obtained from the literature [31-33, 41]. The FCD was distributed as follows [20, 30-32]:

$c_{\mathrm{F}}=-0.1 z^{2}+0.24 z+0.035$

where $z$ is the normalised depth of cartilage ( 0 in top, 1 in bottom). The collagen mass density and fluid fraction were $[20,33,40,41]$ :

$\rho_{\mathrm{c}}=1.4 z^{2}-1.1 z+0.59$,

$n_{\mathrm{f}}=-0.2 z+0.9$

The FCD and fluid fraction of chondrocytes are presented in Table 1, and they were consistent with the previous studies [23, 29, 42, 43].
Table 1 Values of the compositional properties for the 3D cells

\begin{tabular}{lll}
\hline & $\begin{array}{l}\text { Fixed charge } \\
\text { density }(\mathrm{mEq} / \mathrm{ml})\end{array}$ & $\begin{array}{l}\text { Fluid } \\
\text { fraction }(\%)\end{array}$ \\
\hline Superficial zone & 0.10 & 90 \\
Middle zone & 0.13 & 87 \\
Deep zone & 0.17 & 84 \\
\hline
\end{tabular}

\subsection{Simulations}

A total of 8,502 8-node poroelastic continuum elements in one quadrant of the sample were used in the simulations. A total of 360 elements for each PCM and 528 elements for each chondrocyte were used. Chondrocytes were placed in three cartilage zones; superficial zone, middle zone and deep zone. They were assumed to be adhesively attached to the PCM and were therefore fixed to PCM in the geometry. Consequently, continuous displacements and fluid flow in the cell-PCM and PCM-ECM interfaces were assumed [22, $23,26]$. The loading protocol included a free swelling step, a $15 \%$ mechanical compression, and a full relaxation [6].

Boundary conditions were set as follows: (1) movement of the nodes at the bottom of the mesh was prevented in $y$ direction (Fig. 2), (2) symmetry conditions were set on the $x y$-plane and $y z$-plane of the simulated quadrant, (3) free fluid flow was allowed at the edge of the sample, and (4) compression of the sample was applied axially for the top nodes of the mesh. The contacts between the compressing platens and cartilage specimen were assumed frictionless.

The collagen mass fraction, fluid fraction and FCD in the PCM were varied relatively to the composition of the ECM in order to determine their influence on the deformation of cells in different cartilage zones [29]. The finite element simulations were performed using ABAQUS 6.7 (Dassault Systèmes, Providence, RI).

\section{Results}

In the reference configuration, chondrocyte strains and volumes were highly depth-dependent (Fig. 4). Under $15 \%$ static surface-to-surface compression, axial strains of the cells were $11 \%$ (deep zone)-49\% (superficial zone), whereas lateral strains were $2 \%$ (deep zone)-9\% (superficial zone). Strains of the superficial, middle and deep zones of the ECM were also highly depth-dependent, but 1.1-8 times lower than those of the chondrocytes. There were virtually no differences in the cell and ECM strains parallel and perpendicular to the direction of split-lines of the ECM collagen fibrils. Volumes of the superficial, middle and deep zone chondrocytes were 41, 25 and 5\% lower than those before compression, respectively. 

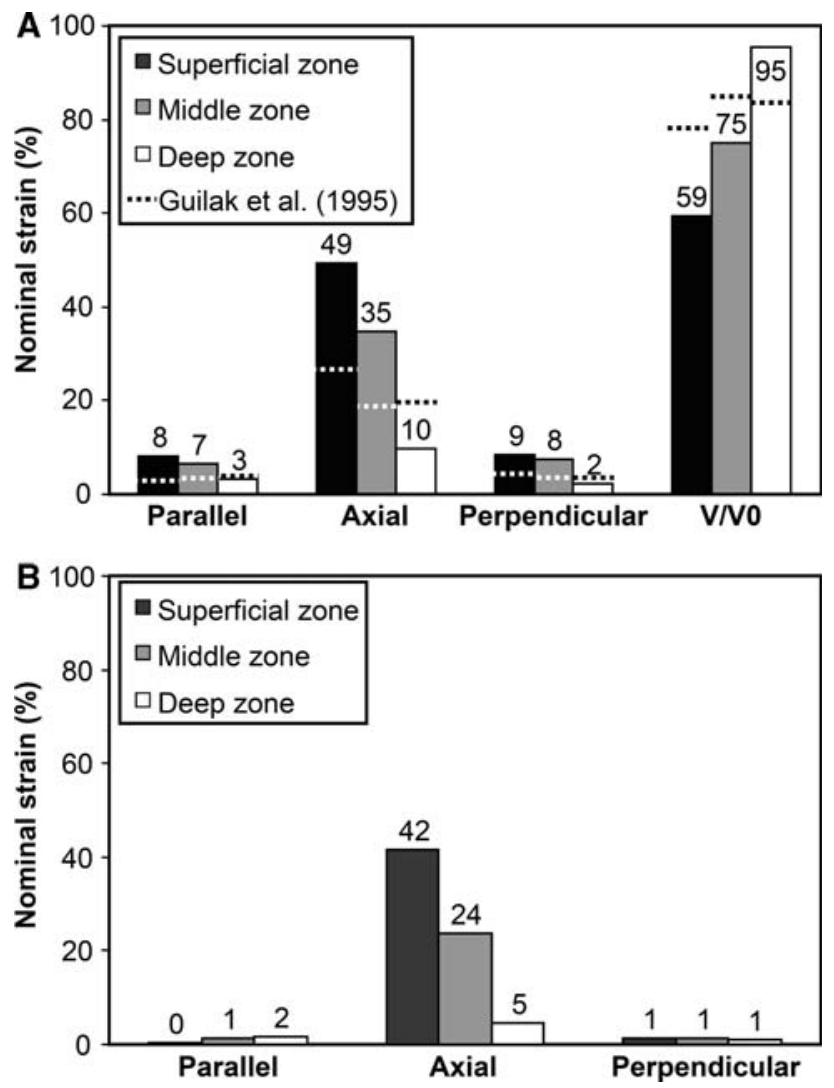

Fig. 4 a Reference axial and lateral (parallel and perpendicular to the split-lines) nominal strains and volume changes (V/V0) for the cells after an application of $15 \%$ surface-to-surface compression of the tissue. b Corresponding axial and lateral strains of the ECM within the proximity of each chondron

Collagen fibril strain distribution $\left(\varepsilon_{\mathrm{f}}\right)$ in the PCM exhibited depth-dependent characteristics (Fig. 5). Lateral edge of the PCM was compressed more in the superficial and middle zone than in the deep zone, therefore, exhibiting low tensile fibril strains, while the fibrils especially in the superficial zone PCM exhibited larger tensile strains above and below the cell. Tensile fibril strains within the PCM were uniform in the deep zone. Fibril strains of the ECM in the proximity of the PCM were lower parallel than perpendicular to the split-lines. Von Mises stresses in the vicinity of the simulated chondrocytes exhibited depthdependent characteristics, greatest stresses being in the superficial zone, and were slightly different parallel and perpendicular to the split-lines (Fig. 6).

The collagen mass fraction, FCD and fluid fraction of the PCMs affected static cell deformation (Table 2) after application of $15 \%$ axial strain for the tissue. An increase in the collagen content of the PCMs was followed by an increase in lateral strains of the superficial and middle zone chondrocytes, whereas the deep zone cell experienced a decrease in lateral strains (Table 2). In the superficial and middle zones, lateral cell strains perpendicular to the split-

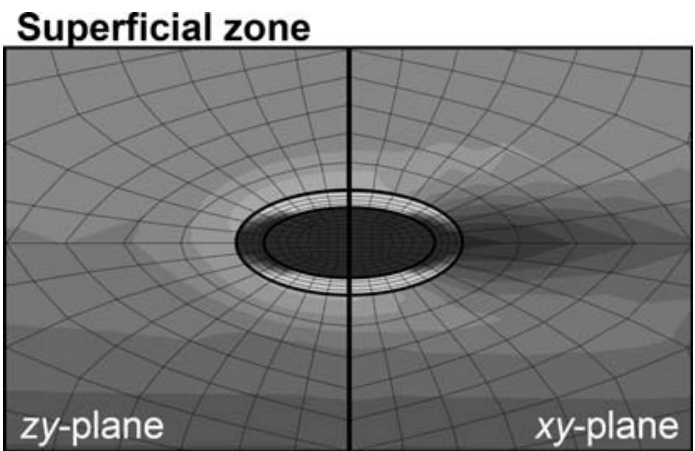

\section{Middle zone}

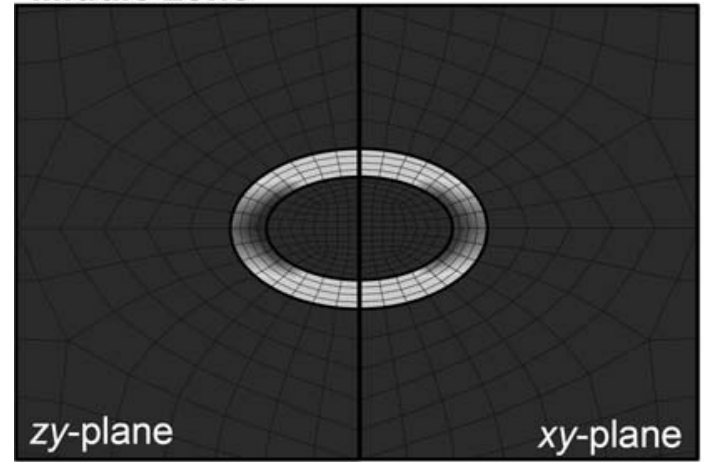

Deep zone
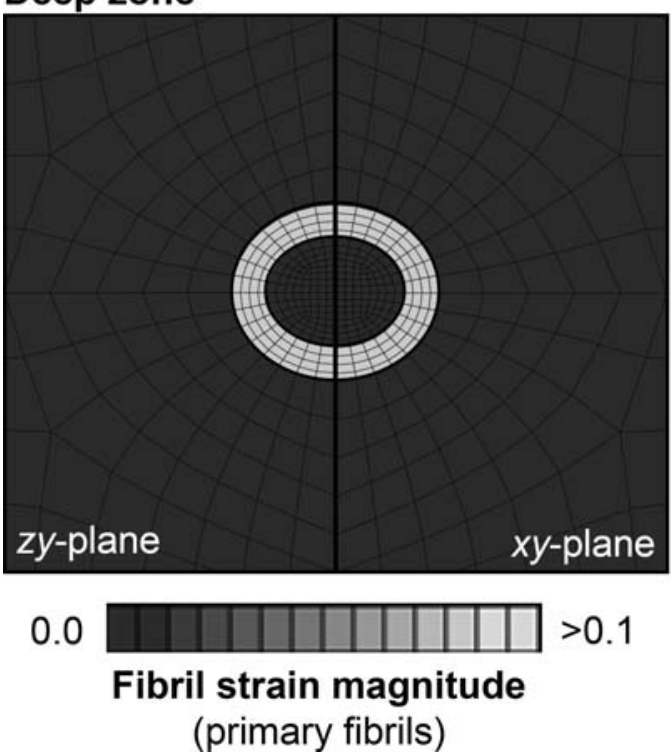

Fig. 5 Distribution of the logarithmic tensile fibril strain of primary collagen fibrils in the proximity of the simulated cells in the reference configuration. Projections in the $x y$ - (parallel to split-lines) and $z y$-planes (perpendicular to split-lines) are presented

line direction were affected more than those parallel to the split-lines after the change in the collagen mass fraction of the PCMs. Axial cell strains were reduced slightly after an increase in the collagen content of the PCMs.

An increase in the pericellular FCD reduced lateral strains of each chondrocyte (Table 2). In the superficial and middle zones, lateral cell strains were reduced more 


\section{Superficial zone}

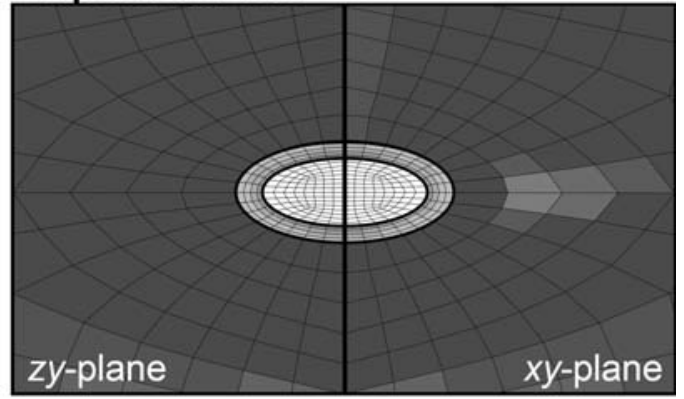

Middle zone

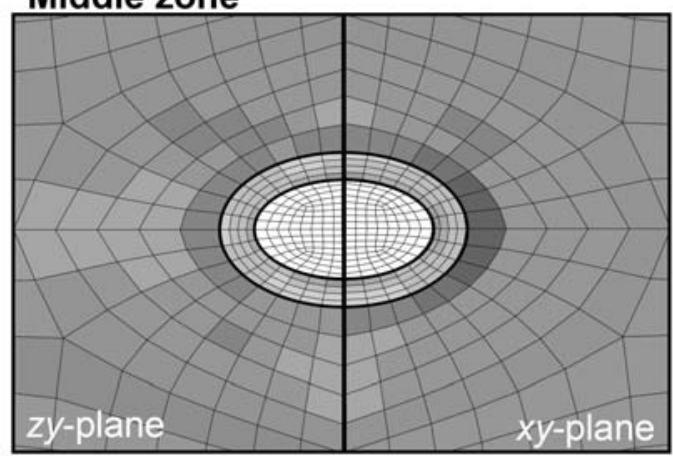

\section{Deep zone}

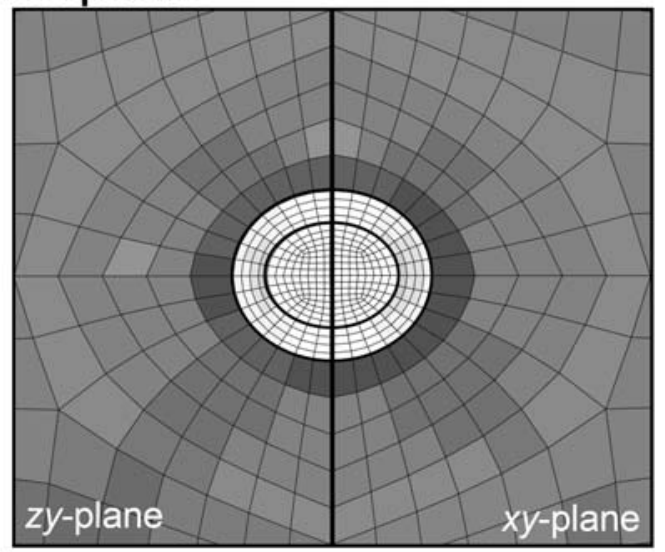

\section{OkPa पप口|⿴囗十 \\ Von Mises stress}

Fig. 6 Von Mises stress distribution in the proximity of the simulated cells in the reference configuration. Projections in the $x y$ - (parallel to split-lines) and zy-planes (perpendicular to split-lines) are presented

parallel than perpendicular to the split-lines as the pericellular FCD was increased. Axial cell strains were only slightly affected by the pericellular FCD.

A decrease in the PCM fluid fraction induced a decrease in lateral strains of the chondrocytes (Table 2). Lateral cell strains changed similarly parallel and perpendicular to the split-lines of the ECM after the change in the pericellular fluid fraction. Axial cell strains were slightly reduced after a decrease in the pericellular fluid fraction.

\section{Discussion}

A 3D finite element model with depth-dependent cartilage properties, including chondrocytes with $\mathrm{PCMs}$ in the superficial, middle and deep zones was constructed in order to study the effect of the PCM composition and the splitlines of the ECM on the cell deformation. Cell deformation was dependent on the collagen and PG content, as well as on the fluid fraction of the PCM. The current model supports earlier found important role of the PCM in modulating the biomechanical signals perceived by the cells $[11,29,44]$, and highlights for the first time the effect of the PCM composition on the mechanical behaviour of chondrocytes. However, one must be careful in comparison between this study and the other studies, as the composition and mechanical properties of the ECM and PCM can be different in these studies.

Axial cell strains were higher in the superficial and middle zone, and lower in the deep zone, as compared to the work by Guilak et al. [6]. On the other hand, lateral cell strains were systematically higher in the superficial and middle zones than those examined by Guilak et al. Lateral cell strains of this study also exhibited clearer depth-dependence. Thereby, also the changes in cell volumes in these studies after static tissue compression were different; $16-22 \%$ in the study by Guilak et al. [6] and $5-41 \%$ in this study. One reason for these differences may be that, due to quite similar axial cell strains and local tissue strains in different cartilage zones, the specimens used by Guilak et al. may have exhibited more homogenous tissue structure and composition than those assumed in our simulations. On the other hand, fluid may have had difficulties to penetrate cell membranes in experimental tests [45], preventing the decrease in cell volumes, whereas we assumed continuous fluid flow through the PCM-cell interface in the simulations. This was a justified assumption because fluid flow should have a negligible effect under equilibrium loading. It is also not certain if 20 min relaxation period was enough for full equilibrium in the experiments by Guilak et al. [6]. At least, a theoretical study by Ateshian et al. [46] showed that it might take much longer time for chondrocytes to reach steady state. In our model, minimal and large changes in the volumes of the superficial and deep zone chondrocytes, respectively, are directly related to the assumed composition and structure of the ECM, obtained from the literature [27]. Obviously, changes in the implemented ECM properties would have affected the deformation behaviour of chondrocytes [22]. However, as the composition, structure and mechanical properties of the simulated samples were unknown, we did not pursue to optimize the correspondence between the experiments and simulations. Thus, the differences between this study 
Table 2 Cell strains parallel $(x)$ and perpendicular $(z)$ to the split-line direction as well as in axial direction $(y)$ in the superficial, middle and deep zones

\begin{tabular}{|c|c|c|c|c|c|c|c|}
\hline \multirow[t]{2}{*}{ Zone } & & \multicolumn{2}{|c|}{ Collagen mass fraction } & \multicolumn{2}{|c|}{ Fixed charge density } & \multicolumn{2}{|l|}{ Fluid fraction } \\
\hline & & $0.1 \times \mathrm{ECM}$ & $1.5 \times \mathrm{ECM}$ & $0.5 \times \mathrm{ECM}$ & $1.3 \times \mathrm{ECM}$ & $0.75 \times \mathrm{ECM}$ & $1.05 \times \mathrm{ECM}$ \\
\hline \multirow[t]{3}{*}{ Superficial } & $x(\%)$ & 7.5 & 8.3 & 9.0 & 7.2 & 8.6 & 9.8 \\
\hline & $y(\%)$ & 50.0 & 48.8 & 48.7 & 50.1 & 47.9 & 48.7 \\
\hline & $z(\%)$ & 7.1 & 8.9 & 8.8 & 7.6 & 8.9 & 9.7 \\
\hline \multirow[t]{3}{*}{ Middle } & $x(\%)$ & 6.3 & 6.5 & 8.1 & 6.9 & 6.7 & 7.5 \\
\hline & $y(\%)$ & 34.9 & 34.3 & 34.2 & 34.3 & 30.1 & 34.7 \\
\hline & $z(\%)$ & 7.0 & 7.7 & 8.4 & 8.2 & 7.6 & 8.3 \\
\hline \multirow[t]{3}{*}{ Deep } & $x(\%)$ & 3.7 & 3.1 & 5.3 & 3.2 & 2.5 & 3.4 \\
\hline & $y(\%)$ & 10.8 & 8.5 & 9.2 & 8.6 & 6.2 & 10.1 \\
\hline & $z(\%)$ & 2.7 & 2.3 & 4.4 & 2.3 & 1.8 & 2.5 \\
\hline
\end{tabular}

Strains were calculated with different values of PCM composition

and the study by Guilak et al. were expected. By optimizing the model parameters, the correspondence would have likely been better, but the optimizing solution would not have been unique due to too many optimized parameters with respect to the experimental data.

Recently, in situ indentation studies were conducted to investigate the deformation behaviour of superficial zone chondrocytes of rabbit articular cartilage [47]. Han and Herzog [47] suggested that the superficial zone chondrocytes experience greater strains along the split-line direction than perpendicular to the split-lines. This behaviour was also observed with some material parameter choices of the present model (e.g. with low collagen content and FCD). Han and Herzog found lower axial cell strains as was simulated here. However, the comparison between these studies may be meaningless since the measurement geometries were different (unconfined versus indentation). Moreover, similarly as in the comparison of this study and Guilak et al. [6], tissue composition and structure may have been significantly different in Han and Herzog [47] and this study, affecting significantly the cell deformations.

In the presented model, we assumed that the cells were initially round in each zone of the cartilage. However, it is known that chondrocytes are ellipsoidal in the superficial zone of cartilage [6, 48]. Reasons for the flatness of the cells are not known. Our previous study showed that initially round cells became ellipsoidal in the superficial zone after the free swelling step $(0.15 \mathrm{M}$, physiological saline solution) prior to compression [26]. This observation was also made in this study. Initially spherical cell shape has also been assumed in several earlier cell level models of articular cartilage [26, 42, 49-51]. Further, it has earlier been suggested that the modelling cells initially ellipsoidal instead of spherical could slightly increase cell strains [52], indicating that our choice for the cell shape resulted in a better correspondence between the model and experiments than that would have been with initially ellipsoidal superficial zone cell.

An increase in the collagen content of the PCMs enhanced lateral cell strain in the superficial zone cell, while the lateral cell strain of the deep zone cell decreased as the collagen content increased. Consistent with our previous study [26], these differences were due to the Benninghoff-type collagen orientation. The increase in the collagen content increased the stiffness of the collagen, which induced increased cell strain in the superficial zone where the fibrils were oriented parallel to the cartilage surface. In contrast, the vertically oriented fibrils in the deep zone restricted the cell expansion more effectively as the collagen content increased.

A decrease in the FCD of the PCM increased lateral strains of the cells. This was due to softening of the cell surroundings, allowing greater local deformation for the PCM. Moreover, stresses of the collagen fibrils were reduced as a result of the loss in the FCD, amplifying local PCM strains especially in the direction parallel to the splitlines. The decrease in the fluid fraction of the PCMs, which increased the PCM stiffness, induced similar changes in the cell strains as the increase in the FCD.

It was suggested earlier that the fluid fraction of chondrocytes may be much lower than what was assumed in this study $[34,50]$. Also, other cell properties in the model were estimated. Therefore, we performed an additional parametric study to test the effect of cell properties, i.e., solid matrix shear modulus, water fraction and FCD, on cell deformations. Decreasing the water fraction by $30 \%$ increased lateral strains by $1-3 \%$ (deep to superficial zone) and decreased axial strains by $0-6 \%$ (deep to superficial zone). Decreasing the FCD by $30 \%$ increased lateral strains by 1-3\% (deep to superficial 
zone) and increased axial strains by $2-7 \%$ (deep to superficial zone). The effect of the shear modulus on the deformation behaviour of the cells was much smaller than that of the water fraction or FCD. The noticed effects were rather similar in the $x y$ - and $z y$-planes.

It is likely that the ratio between the ECM and PCM stiffness is depth-dependent. This is supported by the depth-dependent stiffness of the ECM and depth-independent stiffness of the PCM $[35,53]$. Further, the collagen arrangement in the PCM remains rather similar through tissue depth [7], while the ECM has a unique depth-wise collagen organization [14]. The collagen network of the PCM and ECM together with the depthdependent PG molecules were accounted for in the present model. These parameters together determine the experimentally measured ECM and PCM stiffness in equilibrium. Thus, the ECM/PCM stiffness ratio of the model varied thought the tissue depth. Moreover, the parametric analysis of the effect of different collagen content and FCD of the PCM induced several different ECM/PCM stiffness ratios. Changes in the ECM composition or stiffness, and consequently ECM/PCM stiffness ratio, would of course have had an impact on the outcome of the simulations. However, it was already a subject of our earlier study [26].

This study was is the first study to incorporate a 3D fibril-reinforced model with swelling properties for the ECM and PCM together with chondrocytes embedded into the model. Applying the composition-based model allowed the estimation of the effects of the collagen content, FCD and fluid fraction of the PCM on the deformation behaviour of cells in articular cartilage. This study was focused on investigating articular cartilage and cells under static loading. This loading configuration has been shown to inhibit aggrecan biosynthesis [54]. On the contrary, dynamic loading has been shown to stimulate proteoglycan synthesis [55]. In order to clarify the impact of cell mechanics on cartilage biosynthesis, the relationships between mechanical and biological responses of cells should be investigated in the future in static and dynamic loading configuration. For that, the number of optimized model parameters should be reduced, i.e., as many compositional, structural and mechanical parameters of the ECM, PCM and cells as possible should be measured experimentally (e.g. [17, 56, 57]) and implemented in the model. Following that, cell deformations should be compared with experimental measurements of cells in situ under mechanical and/or osmotic loading [47], and the rest of the parameters in the PCM and cells could be possibly optimized. This could lead to a samplespecific estimation of stress and strain environment of chondrocytes.

\section{Conclusions}

In conclusion, based on a microscopic 3D model of articular cartilage, collagen and PGs of the PCM influence the mechanical signals detected by the chondrocytes differently. Hence, their effect may be different for the cartilage biosynthesis. Decrease in the collagen content and increase in the pericellular FCD reduced lateral cell strains which might highlight the protective role of the PCM [58]. Furthermore, the collagen content affected lateral cell strains in the superficial and middle zone more perpendicular than parallel to split-line direction, while with FCD, the trend was opposite. However, cartilage is a very complex tissue and the interplay between collagen, PGs and fluid ultimately modulate the mechanical environment of chondrocytes. The present model may improve the understanding of the significance of the chondrocyte environment on the degeneration, adaptation and remodelling of articular cartilage. We believe that this information can be used to estimate the impact of the structural and compositional changes in PCMs (e.g., during osteoarthrosis) on the maintenance of articular cartilage integrity.

Acknowledgements Funding from the Academy of Finland (Project No. 125415); Alberta Heritage Foundation for Medical Research, Alberta, Canada; Sigrid Juselius Foundation, Finland; Kuopio University Hospital, Kuopio, Finland (EVO); Biomaterial Graduate School, Finland; Emil Aaltonen Foundation, Finland; and Finnish Cultural Foundation of Northern Savo is acknowledged. CSC-IT Center for Science is acknowledged for technical support.

Open Access This article is distributed under the terms of the Creative Commons Attribution Noncommercial License which permits any noncommercial use, distribution, and reproduction in any medium, provided the original author(s) and source are credited.

\section{References}

1. Bachrach NM, Valhmu WB, Stazzone E et al (1995) Changes in proteoglycan synthesis of chondrocytes in articular cartilage are associated with the time-dependent changes in their mechanical environment. J Biomech 28:1561-1569

2. Buschmann MD, Gluzband YA, Grodzinsky AJ et al (1995) Mechanical compression modulates matrix biosynthesis in chondrocyte/agarose culture. J Cell Sci 108:1497-1508

3. Trickey WR, Vail TP, Guilak F (2004) The role of the cytoskeleton in the viscoelastic properties of human articular chondrocytes. J Orthop Res 22:131-139

4. Poole A R, Alini M, Hollander A P (1995) Cellular biology of cartilage degradation. In: Mechanism and models in rheumatoid arthritis. Academic Press Ltd, London, pp 163-204

5. Guilak F, Ratcliffe A, Lane N et al (1994) Mechanical and biochemical changes in the superficial zone of articular cartilage in canine experimental osteoarthritis. J Orthop Res 12:474-484

6. Guilak F, Ratcliffe A, Mow VC (1995) Chondrocyte deformation and local tissue strain in articular cartilage: a confocal microscopy study. J Orthop Res 13:410-421 
7. Kaab MJ, Richards RG, Ito K et al (2003) Deformation of chondrocytes in articular cartilage under compressive load: a morphological study. Cells Tissues Organs 175:133-139

8. Wilson W, Driessen N, van Donkelaar C et al (2006) Mechanical regulation of the chondron collagen fiber network structure. Trans Orthop Res Soc 31:1520

9. Poole CA, Ayad S, Schofield JR (1988) Chondrons from articular cartilage: I. Immunolocalization of type VI collagen in the pericellular capsule of isolated canine tibial chondrons. J Cell Sci 90:635-643

10. Poole CA, Honda T, Skinner SJ et al (1990) Chondrons from articular cartilage (II): analysis of the glycosaminoglycans in the cellular microenvironment of isolated canine chondrons. Connect Tissue Res 24:319-330

11. Alexopoulos LG, Williams GM, Upton ML et al (2005) Osteoarthritic changes in the biphasic mechanical properties of chodrocyte pericellular matrix in articular cartilage. J Biomech 38:509-517

12. Guilak F, Jones WR, Ting-Beall HP et al (1999) The deformation behavior and mechanical properties of chondrocytes in articular cartilage. Osteoarthr Cartil 7:59-70

13. Korhonen RK, Herzog W (2008) Depth-dependent analysis of the role of collagen fibrils, fixed charges and fluid in the pericellular matrix of articular cartilage on chondrocyte mechanics. J Biomech 41:480-485

14. Speer DP, Dahners L (1979) The collagenous architecture of articular cartilage. Correlation of scanning electron microscopy and polarized light microscopy observations. Clin Orthop 139:267-275

15. Julkunen P, Kiviranta P, Wilson W et al (2007) Characterization of articular cartilage by combining microscopic analysis with a fibril-reinforced finite-element model. J Biomech 40:1862-1870

16. Julkunen P, Korhonen RK, Nissi MJ et al (2008) Mechanical characterization of articular cartilage by combining magnetic resonance imaging and finite-element analysis: a potential functional imaging technique. Phys Med Biol 53:2425-2438

17. Julkunen P, Wilson W, Jurvelin JS et al (2008) Stress-relaxation of human patellar articular cartilage in unconfined compression: prediction of mechanical response by tissue composition and structure. J Biomech 41:1978-1986

18. Li LP, Cheung JT, Herzog W (2009) Three-dimensional fibrilreinforced finite element model of articular cartilage. Med Biol Eng Comput 47:607-615

19. Shirazi R, Shirazi-Adl A, Hurtig M (2008) Role of cartilage collagen fibrils networks in knee joint biomechanics under compression. J Biomech 41:3340-3348

20. Wilson W, Huyghe JM, van Donkelaar CC (2007) Depthdependent compressive equilibrium properties of articular cartilage. Biomech Model Mechanobiol 6:43-53

21. Guilak F, Erickson GR, Ting-Beall HP (2002) The effects of osmotic stress on the viscoelastic and physical properties of articular chondrocytes. Biophys J 82:720-727

22. Korhonen RK, Julkunen P, Rieppo J et al (2006) Collagen network of articular cartilage modulates fluid flow and mechanical stresses in chondrocyte. Biomech Model Mechanobiol 5:150-159

23. Likhitpanichkul M, Guo XE, Mow VC (2005) The effect of matrix tension-compression nonlinearity and fixed negative charges on chondrocyte responses in cartilage. MCB 2:191-204

24. Trickey WR, Baaijens FP, Laursen TA et al (2006) Determination of the Poisson's ratio of the cell: recovery properties of chondrocytes after release from complete micropipette aspiration. J Biomech 39:78-87

25. Trickey WR, Lee GM, Guilak F (2000) Viscoelastic properties of chondrocytes from normal and osteoarthritic human cartilage. J Orthop Res 18:891-898
26. Korhonen RK, Julkunen P, Wilson W et al (2008) Importance of collagen orientation and depth-dependent fixed charge densities of cartilage on mechanical behavior of chondrocytes. J Biomech Eng 130:021003

27. Wilson W, Huyghe JM, van Donkelaar CC (2006) A composition-based cartilage model for the assessment of compositional changes during cartilage damage and adaptation. Osteoarthr Cartil 14:554-560

28. Guilak F (2000) The deformation behavior and viscoelastic properties of chondrocytes in articular cartilage. Biorheology 37:27-44

29. Guilak F, Mow VC (2000) The mechanical environment of chondrocyte: a biphasic finite element model of cell-matrix interactions in articular cartilage. J Biomech 33:1663-1673

30. Chen SS, Falcovitz YH, Schneiderman R et al (2001) Depthdependent compressive properties of normal aged human femoral head articular cartilage: relationship to fixed charge density. Osteoarthr Cartil 9:561-569

31. Maroudas A (1968) Physicochemical properties of cartilage in the light of ion exchange theory. Biophys J 8:575-595

32. Maroudas A, Bannon C (1981) Measurement of swelling pressure in cartilage and comparison with the osmotic pressure of constituent proteoglycans. Biorheology 18:619-632

33. Rieppo J, Hyttinen MM, Lappalainen R et al (2004) Spatial determination of water, collagen and proteoglycan contents by Fourier Transform Infrared imaging and digital densitometry. Trans Orthop Res Soc 29:1021

34. Mow VC, Kuei SC, Lai WM et al (1980) Biphasic creep and stress relaxation of articular cartilage in compression: theory and experiments. J Biomech Eng 102:73-84

35. Guilak F, Alexopoulos LG, Haider MA et al (2005) Zonal uniformity in mechanical properties of the chondrocyte pericellular matrix: micropipette aspiration of canine chondrons isolated by cartilage homogenization. Ann Biomed Eng 33:1312-1318

36. Poole CA, Ayad S, Gilbert RT (1992) Chondrons from articular cartilage. V. Immunohistochemical evaluation of type VI collagen organisation in isolated chondrons by light, confocal and electron microscopy. J Cell Sci 103:1101-1110

37. Wilson W, van Donkelaar CC, van Rietbergen B et al (2005) A fibril-reinforced poroviscoelastic swelling model for articular cartilage. J Biomech 38:1195-1204

38. Huyghe JM, Houben GB, Drost MR et al (2003) An ionised/nonionised dual porosity model of intervertebral disc tissue. Biomech Model Mechanobiol 2:3-19

39. Basser PJ, Schneiderman R, Bank RA et al (1998) Mechanical properties of the collagen network in human articular cartilage as measured by osmotic stress technique. Arch Biochem Biophys 351:207-219

40. Shapiro EM, Borthakur A, Kaufman JH et al (2001) Water distribution patterns inside bovine articular cartilage as visualized by $1 \mathrm{H}$ magnetic resonance imaging. Osteoarthr Cartil 9:533-538

41. Lipshitz H, Etheredge Rd, Glimcher MJ (1975) In vitro wear of articular cartilage. J Bone Joint Surg Am 57:527-534

42. Lai WM, Sun DD, Ateshian GA et al (2002) Electrical signals for chondrocytes in cartilage. Biorheology 39:39-45

43. Schugart RC (2005) Mathematical models and numerical methods for analysis of mechanical and chemical loading in articular cartilage. North Carolina State University, Raleigh

44. Hing WA, Sherwin AF, Poole CA (2002) The influence of the pericellular microenvironment on the chondrocyte response to osmotic challenge. Osteoarthr Cartil 10:297-307

45. Xu X, Cui Z, Urban JP (2003) Measurement of the chondrocyte membrane permeability to Me2SO, glycerol and 1, 2-propanediol. Med Eng Phys 25:573-579 
46. Ateshian GA, Costa KD, Hung CT (2007) A theoretical analysis of water transport through chondrocytes. Biomech Model Mechanobiol 6:91-101

47. Han S-K, Herzog W (2007) Novel In Situ Chondrocyte Indentation Study. J Biomech 40(Suppl 2):S39

48. Choi JB, Youn I, Cao L et al (2007) Zonal changes in the threedimensional morphology of the chondron under compression: the relationship among cellular, pericellular, and extracellular deformation in articular cartilage. J Biomech 40:2596-2603

49. Alexopoulos LG, Setton LA, Guilak F (2005) The biomechanical role of the chondrocyte pericellular matrix in articular cartilage. Acta Biomater 1:317-325

50. Wu JZ, Herzog W (2000) Finite element simulation of locationand time-dependent mechanical behavior of chondrocytes in unconfined compression tests. Ann Biomed Eng 28:318-330

51. Wu JZ, Herzog W (2006) Analysis of the mechanical behavior of chondrocytes in unconfined compression tests for cyclic loading. J Biomech 39:603-616

52. Chahine NO, Hung CT, Ateshian GA (2007) In situ measurements of chondrocyte deformation under transient loading. Eur Cell Mater 13:100-111
53. Schinagl RM, Gurskis D, Chen AC et al (1997) Depth-dependent confined compression modulus of full-thickness bovine articular cartilage. J Orthop Res 15:499-506

54. Quinn TM, Grodzinsky AJ, Buschmann MD et al (1998) Mechanical compression alters proteoglycan deposition and matrix deformation around individual cells in cartilage explants. J Cell Sci 111(Pt 5):573-583

55. Buschmann MD, Kim YJ, Wong M et al (1999) Stimulation of aggrecan synthesis in cartilage explants by cyclic loading is localized to regions of high interstitial fluid flow. Arch Biochem Biophys 366:1-7

56. Alexopoulos LG, Haider MA, Vail TP et al (2003) Alterations in the mechanical properties of the human chondrocyte pericellular matrix with osteoarthritis. J Biomech Eng 125:323-333

57. Ng L, Hung HH, Sprunt A et al (2007) Nanomechanical properties of individual chondrocytes and their developing growth factor-stimulated pericellular matrix. J Biomech 40:1011-1023

58. Poole CA, Flint MH, Beaumont BW (1988) Chondrons extracted from canine tibial cartilage: preliminary report on their isolation and structure. J Orthop Res 6:408-419 